\title{
Eine transportable Motor-Durchlaufwinde (Winch) zum Einsatz in beliebigen Booten
}

\begin{abstract}
A transportable powered winch for use in any boat

A simple, transportable powered winch which can be mounted in any boat is described. It.is operated by a 200-watt geared electromotor. The lifting power at the capstan amounts to $50 \mathrm{kp}$ at a lifting speed of $0.4 \mathrm{~m} / \mathrm{s}$. The load can be pivoted into the boat by a small crane integrated in the machinery. For special uses such as rapid hoisting of lighter measuring probes, rewinding of the supporting cable, etc., the capstan can be exchanged for any type of cable drums. The current is provided by a 220 -volt transportable electric generator.
\end{abstract}

Das Bedürfnis nach geeigneten Hebezeugen, Kranen, Winden usw. dürfte annähernd so alt sein wie die limnologische Feldarbeit. Während bei küsten- und hochseetüchtigen Schiffen derartige Einrichtungen notwendig und alltäglich sind, sind sie beim Fahrzeugpark auf Binnenseen (mit Ausnahme der Lastschiffe) unbekannt. In der limnologischen Feldarbeit behilft man sich mit kleinen, transportablen handbetriebenen Seilwinden, welche am Boot irgendwie, meist improvisiert, befestigt werden. Nur auf einigen wenigen Arbeitsbooten von Instituten finden sich fest eingebaute leichte Krane und Seilwinden. An derartigen Einrichtungen, welche transportabel, einfach zu befestigen, leicht und trotzdem genügend robust und womöglich auch motorisiert sind, herrscht Mangel.

Im Rahmen eines Programms zur Entwicklung spezifisch limnologischer Probenahmegeräte und darauf abgestimmter Hilfseinrichtungen wurde eine Durchlaufseilwinde zum Heben relativ schwerer Lasten entwickelt. Sie besteht im Prinzip aus einem Spillkopf («capstan»), vergleichbar einer Yachtwinch mit horizontaler Achse. Als Antrieb dient ein 220-V-Kollektor-E-Motor mit $200 \mathrm{~W}$ Leistung, verbunden mit einem Reduktionsgetriebe, dessen Abtriebsachse den Spillkopf trägt (Abb. 1). Solche Getriebemotoren sind in beliebiger Spezifikation, gewissermassen massgeschneidert, erhältlich. Unser Motor ist auf einem Flansch befestigt, der in einem kräftigen Rahmen untergebracht ist. Dieser ist über ein starkes, horizontal gelagertes Gelenk beweglich und erlaubt, einen kleinen Kran mit drehbarer Säule in vertikaler Lage zu fixieren. Die ganze Einrichtung ist auf einem starken .Brett befestigt, welches mit zwei üblichen Schraubzwingen an jeder Bootswand befestigt werden kann (Abb.2). Ist die Bootswand höher als das Brett, so kann dieses mit einem Auszug verlängert werden. Um es auch unten genügend $\mathrm{zu}$ fixieren, wird es mit einem verstellbaren Querstab (Spreize) unmittelbar über dem Fussboden an die Bordwand gedrückt. Auf der Gegenseite wird die Spreize durch ein einfaches Gegenstück, das oben ebenfalls mit einer Schraubzwinge festgehalten wird, fixiert. 

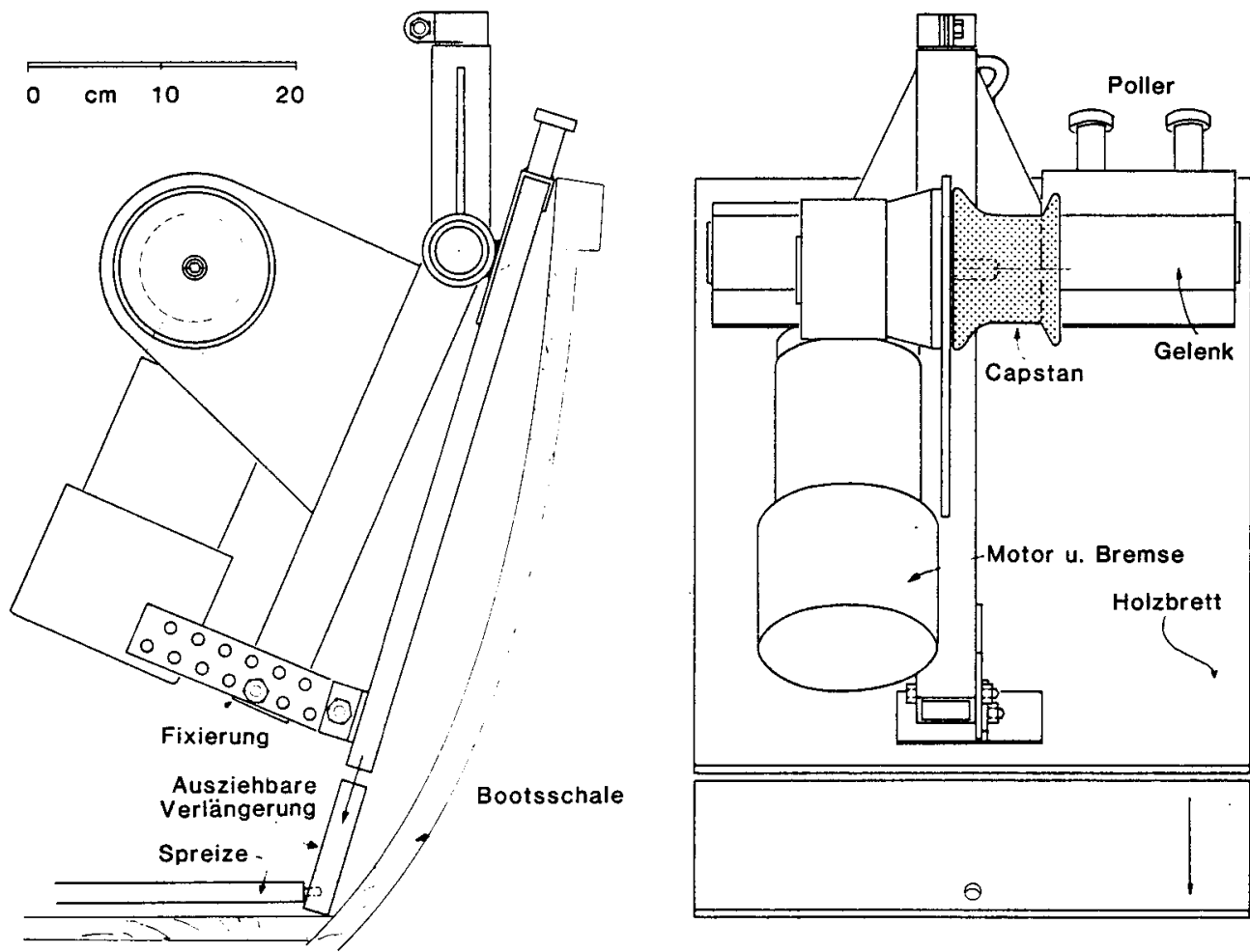

Abb. 1. Konstruktionsskizze. Das Holzbrett kann nach unten verlängert werden. Die Auszugschienen aus Vierkant-Stahlrohr sind nicht gezeichnet, aber in Abb. 3 sichtbar.

Figure 1. Construction plan. The wooden board can be lenghthened at the bottom. The extension rails, maid of square steel piping, are not pictured but can be seen in figure 3 .

Die erwähnte Bauart mit einem handelsüblichen Getriebemotor macht ein besonderes Getriebe oder ein Vorgelege überflüssig. Der Spillkopf (Abb.3) ist mit einem Freilauf versehen, damit bei einer Störung eine bereits hängende Last mit Hilfe einer Handkurbel eingeholt werden kann. Er kann gegen eine Seilspule ausgetauscht werden oder gegen eine Trommel mit dem rund dreifachen Durchmesser, um bei leichteren Lasten eine grössere Hebegeschwindigkeit zu erreichen. Dies ist vor allem für die Bewegung von Unterwasser-Messgeräten mit grossen Kabellängen vorgesehen (Abb.4).

Mit Normalausrüstung (Capstan) hebt die Winde maximal $50 \mathrm{kp}$ mit einer Geschwindigkeit von etwa $0,4 \mathrm{~m} / \mathrm{s}$. Der Strom wird von einem handelsüblichen Generator geliefert. Das Gerät hat sich im harten Einsatz (Heben von Sedimentationsfallen inkl. den Ankern) bewährt. Es ist aus gängigen Materialien (Stahlblech, Stahlrohr, Aluminium) gefertigt und mit handelsüblichem Zubehör wie Seilrollen, Yachtblöcken usw. ausgestattet. Es wurde in der Werkstätte der Versuchsstation Tüffenwies der EAWAG angefertigt. Der Verfasser dankt den Herren W. Häfliger und $\mathrm{E}$. Schwager für ihre ausgezeichnete Konstruktionsarbeit.

H. Ambühl

Adresse des Autors: Prof. H. Ambühl, EAWAG, CH-8600 Dübendorf, Schweiz. 

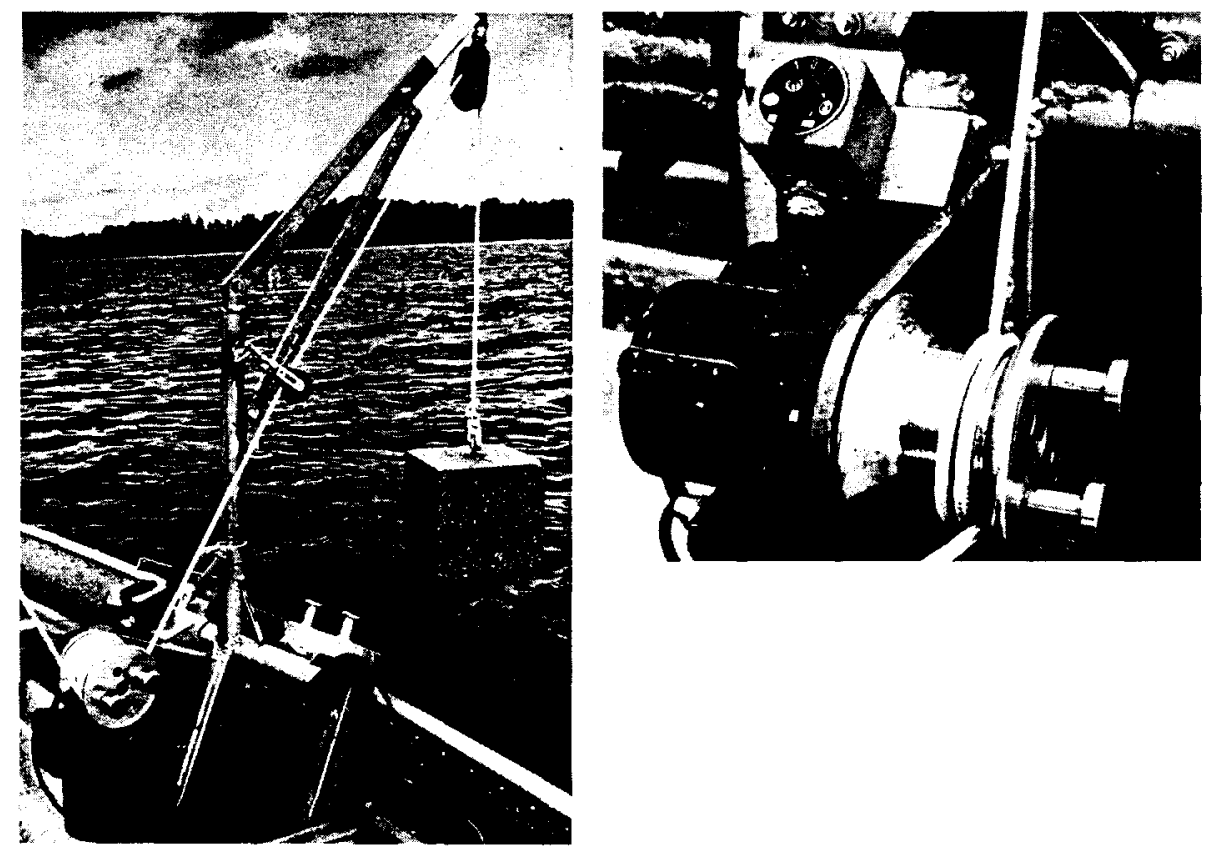

Abb.2. Gesamtansicht. Die hochgezogene Last kann am Kran ins Boot eingeschwenkt werden. Figure 2. Total view. The load on the crane can be pivoted into the boat.

Abb.3. Spillkopf ( «capstan»). Links davon das Schneckengetriebe, oben der Schalter. Figure 3. Capstan. On the left the gear, above the switch.

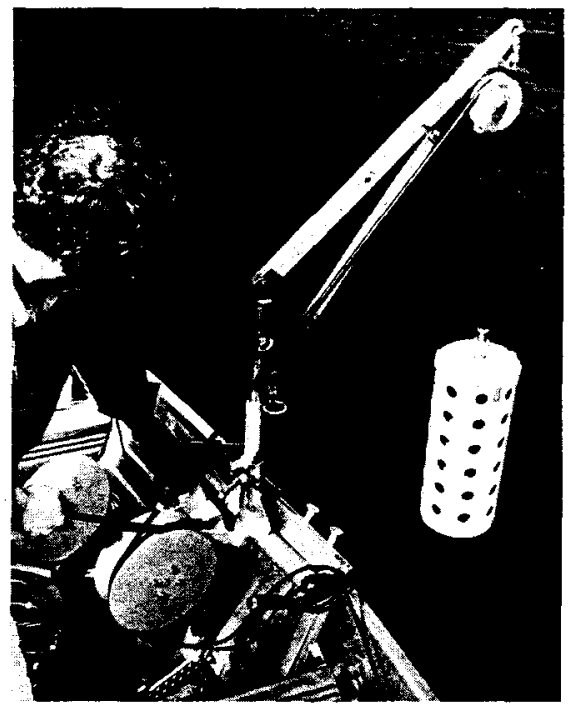

Abb.4. Zum Heben einer Fernmeßsonde mit schwerem Kabel ist der Capstan durch eine grössere Kabeltrommel ersetzt.

Figure 4. To hoist a measuring probe with a heavy cable the capstan is exchanged with a cable drum. 